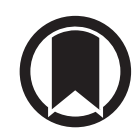

CrossMark

\section{Potential of regulatory T-cell-based therapies in the management of severe COVID-19}

To the Editor:

"Cytokine storm", inflammation-mediated severe lung damage and defective haemostasis are the main underlying reasons for morbidity and mortality in coronavirus disease 2019 (COVID-19) patients [1]. Several immunotherapies that target various inflammatory processes have been successfully used in COVID-19 patients and many other strategies are under evaluation [2, 3]. However, in view of dysregulated immune responses in severe COVID-19 patients, we suggest that $\mathrm{CD} 4{ }^{+} \mathrm{CD} 25^{+}$FoxP $3^{+}$ regulatory T-cell (Treg)-based strategies could be considered for patient management.

Vigorous antimicrobial responses triggered against the pathogen can be detrimental to the host due to collateral tissue damage. Therefore, regulatory mechanisms, and in particular Tregs, are in place to ensure that inflammation is kept in check. Tregs are either thymus-derived or induced in the periphery and are classically known for stimulating immune tolerance, and preventing autoimmune and inflammatory diseases [4]. Tregs inhibit the activation of both innate and adaptive immune cells via inhibitory surface molecules (like cytotoxic T-lymphocyte antigen-4 (CTLA-4) and lymphocyte-activation gene-3) and secretion of immunosuppressive cytokines (interleukin (IL)-10, transforming growth factor- $\beta$ and IL-35). Both Treg subsets are equally important to prevent inflammation-induced tissue damage during acute infections and to promote tissue repair, which is particularly shown in the influenza infection model $[4,5]$.

The current evidence suggests that the level of peripheral Tregs is prominently reduced in severely ill COVID-19 patients compared with mild patients [6-9]. Though the reasons for reduced frequency of Tregs in peripheral blood are not completely understood, one of the possibilities is that Tregs might have migrated to the lungs to prevent tissue damage. Detailed investigation of Tregs in the lung tissues of severe COVID-19 patients and their molecular signatures would provide insight on these questions. However, in silico analyses performed on the transcriptomic data of $\mathrm{CD}^{+}$T-cells from the COVID-19 patient's bronchoalveolar lavage suggest that IL2 transcripts were reduced in severe cases compared with mild cases [10]. Therefore, reduced IL2 would lead to enhanced apoptosis of Tregs and is also confirmed by reduced levels of FoxP3. Moreover, severe COVID-19 patients have increased levels of soluble IL-2R (CD25) [6-8], probably due to inflammation-induced enhanced proteolytic cleavage of cell surface CD25. This soluble CD25 could potentially interfere with IL-2 bioavailability and signalling, and hence might further promote apoptosis of Tregs. It has also been shown that Middle East respiratory syndrome coronavirus could infect T-cells [11] and hence, a direct effect of severe acute respiratory syndrome coronavirus 2 (SARS-CoV-2) on the biology of Tregs cannot be ruled out.

Considering the importance of Tregs in immune homeostasis, reduction in the levels of Tregs could be one of the reasons for the hyperactivated immune system and damaged lungs in severe COVID-19 patients. It is worth noting that depletion of Treg from the mice infected with murine coronavirus lead to increased mortality with acute encephalitis, thus highlighting the protective nature of Tregs during acute coronavirus infection [12]. It should also be noted that obesity is one of the risk factors for COVID-19, and data from obese subjects and pertinent animal models have shown that Tregs in the circulation and visceral adipose tissues are decreased compared with those in lean subjects, and consequently there is a higher state of inflammation and insulin resistance $[13,14]$.

@ERSpublications

In view of dysregulated immune response, "cytokine storm" and inflammation-induced lung damage in severely ill COVID-19 patients, we propose that $\mathrm{CD}^{+} \mathrm{CD}^{+} 5^{+} \mathrm{FoxP} 3^{+}$regulatory $\mathrm{T}$-cell-based therapies could be considered for patient management https://bit.ly/3eKqWPo

Cite this article as: Stephen-Victor E, Das M, Karnam A, et al. Potential of regulatory T-cell-based therapies in the management of severe COVID-19. Eur Respir J 2020; 56: 2002182 [https://doi.org/ $10.1183 / 13993003.02182-2020]$. 
Given the dysregulated immune response in severe COVID-19 patients, we propose that Tregs have therapeutic potential in patient management. Adoptive transfer of ex vivo expanded polyclonal Tregs has recently been used to treat autoimmune and inflammatory diseases [15]. But polyclonal Treg therapy is time consuming, requiring nearly 2 weeks to expand sufficient quantities of viable clinical-grade Tregs for immunotherapy. However, unlike autoimmune diseases, COVID-19 patients need an instant therapy to prevent morbidity and mortality. Therefore, adoptive transfer autologous polyclonal Treg therapy is not a viable option in COVID-19 patients. Also, the approach is not economically feasible for an infectious disease. Alternatively, allogeneic human leukocyte antigen-matched umbilical cord-derived Tregs are under exploration for inflammatory conditions (ongoing clinical trials registered at ClinicalTrials.gov: NCT02932826 and NCT03011021) (figure 1) [16]. In view of the drawbacks of autologous Treg therapy, allogeneic-matched cord Tregs could be considered for severe COVID-19 patients. Of late, two COVID-19 patients with acute respiratory distress syndrome (ARDS) were treated twice or thrice with allogeneic, offthe-shelf, cord-blood derived, ex vivo expanded Tregs $\left(1 \times 10^{8}\right.$ cells per dose). The Treg therapy was associated with rapid decline in the various inflammatory mediators including IL-6, TNF- $\alpha$, IFN- $\gamma$, IL- 8 and IL-12 [17].

Another strategy is to boost Tregs in vivo. Low-dose IL-2 has been used to specifically induce Treg expansion in vivo in type 1 diabetes, and other autoimmune and inflammatory diseases [18]. The high affinity of CD25 towards IL-2 would lead to selective Treg expansion. But severe COVID-19 patients display increased levels of soluble IL-2R [6-8] that could potentially scavenge IL-2. Other reports have demonstrated that severe COVID-19 patients have increased IL-2 levels [1] and despite having higher IL-2, Tregs were lower in severe COVID-19 patients. All these arguments suggest that low-dose IL-2 therapy might not be beneficial in COVID-19 patients. Nevertheless, a clinical trial is planned with low-dose IL-2 for acute respiratory distress syndrome related to COVID-19 (trial NCT04357444 registered at ClinicalTrials.gov). Data from this trial will provide valuable information on the feasibility of this strategy not only for COVID-19 but also for other acute viral diseases. Other Treg expansion strategies, like IL-2 complexed with monoclonal antibodies to selectively trigger Treg activation [19] or to induce STAT5 phosphorylation and Treg expansion [20], have been explored in pre-clinical models. But they have yet to be tested in clinic and in view of their unproven efficacy in patients, it is too risky to use them in COVID-19 patients.
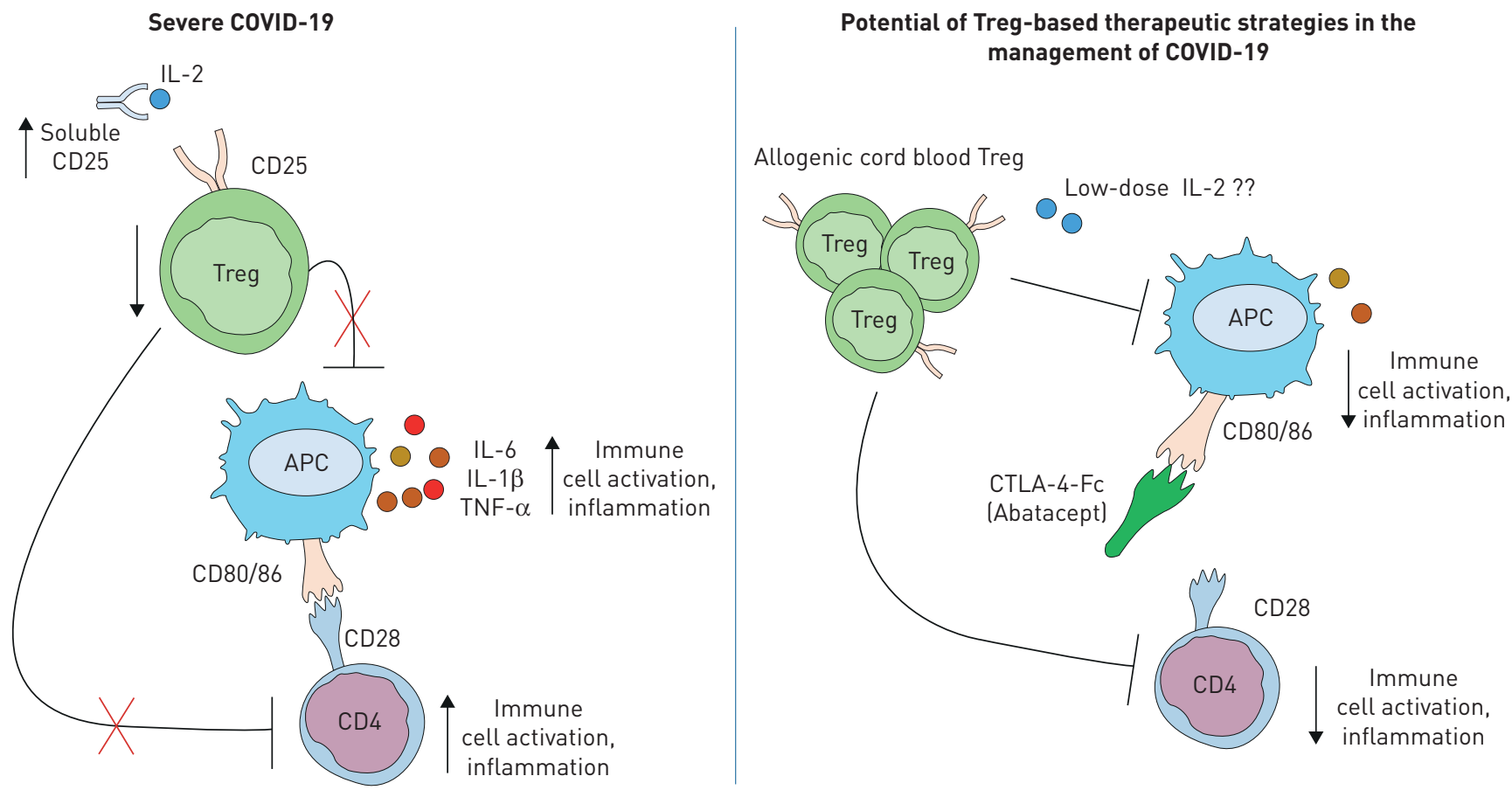

FIGURE 1 Potential of regulatory T-cell (Treg)-based therapies in the management of severe coronavirus disease 2019 (COVID-19). Tregs and their functions are compromised in severe COVID-19 patients, engendering unrestrained immune cell activation. Dysregulated antigen-presenting cells (APCs) insinuate tissue inflammation and immunopathology by secreting inflammatory cytokines and activating a T-cell-dependent immune response. We suggest that either adoptive transfer of allogenic Tregs or use of Treg-derived molecules like cytotoxic T-lymphocyte antigen (CTLA)-4 (abatacept) might block the activation of APC and costimulatory pathways. Such therapies have the potential to curtail tissue inflammation and immunopathology, leading to better management of COVID-19 patients. IL: interleukin; TNF: tumour necrosis factor. 
In view of various shortcomings with either adoptive Treg therapy or in vivo Treg expansion strategies, therapy with Treg-derived immunoregulatory molecules, in particular CTLA-4, might hold the potential for controlling inflammation in severe COVID-19 patients (figure 1). At cellular level, CTLA-4 interacts with B7 family members (CD80 and CD86) on innate cells. Through trans-endocytosis and degradation of B7 molecules inside CTLA-4-expressing cells, CTLA-4 reduces co-stimulatory signals for T-cells [21]. The recombinant Fc-fused CTLA-4 protein abatacept has been used for several years for the immunotherapy of systematic autoimmune diseases. Though our knowledge on the mechanisms of this fusion protein is still incomplete, current literature suggest that by interacting with B7 molecules on antigen-presenting cells, abatacept interferes with CD28-mediated T-cell signalling and activation [22]. Abatacept might also affect the activation of innate cells, such as monocytes and dendritic cells, and enhance Tregs, though data on the Treg function are limited and conflicting [23, 24].

While data is not available regarding abatacept therapy in COVID-19 patients, recent longitudinal observational studies on the incidence of COVID-19 in abatacept-treated patients provide a pointer towards its therapeutic potential in severely ill COVID-19 patients. An epidemiological survey performed in the large tertiary hospital in Barcelona (Spain) indicated that abatacept-treated patients (42 patients from a cohort of 959 patients treated with biological and synthetic disease-modifying anti-rheumatic drugs (DMARD)) exhibited the lowest frequency of COVID-19-compatible symptoms [25]. Similar data were also obtained from a hospital in Madrid (Spain), where it was noted that none of the 27 abatacept-treated patients (among 802 DMARD-treated patients) were admitted to hospital with COVID-19 symptoms [26]. Furthermore, among 779 biological DMARD-treated patients from Siena (Italy), only two tested positive for COVID-19 and notably none of the 55 abatacept-treated patients in this cohort experienced COVID-19 [27].

Although these observations are from small cohorts and it is possible that the patients were practicing strict social distancing, the data on abatacept-treated patients are not surprising in view of its documented clinical benefits in autoimmune diseases [28] and the mechanisms of action of CTLA-4 to mitigate inflammatory responses.

Various immunotherapies that target the cytokine storm and prevent lung damage have gained prominence in the management of severe COVID-19 patients. Among the Treg-based therapeutic approaches, CTLA-4-based therapies appear to be attractive to overcome the hyperinflammatory state of severely ill COVID-19 patients. Clinicaltrials.gov registry shows that 47 studies are registered for the evaluation of tocilizumab (a humanised monoclonal antibody to IL-6 receptor $\alpha$ )/sarilumab (a fully human monoclonal antibody to IL-6 receptor $\alpha$ ) in COVID-19 patients and 14 studies are registered for anakinra (an IL-1 receptor antagonist). However, just a single Phase 2 clinical trial has been registered recently for abatacept therapy in COVID-19 patients (trial NCT04472494 registered at ClinicalTrials.gov). Initial clinical trials mainly focused on the management of the cytokine storm and in particular, the role of IL-6 and IL-1 $\beta$. Data on the Tregs in COVID-19 patients were also scarce during the early period of the COVID-19 pandemic. These points may explain why abatacept is yet to be considered for the management of COVID-19 patients, apart from the recently registered clinical trial (NCT04472494). As more data emerge on the fate of Tregs in COVID-19 patients, the aforementioned clinical observations could be taken as a basis for initiating randomised trials on CTLA-4-Fc (abatacept) therapy in moderate or severe COVID-19 patients. Though abatacept therapy in autoimmune patients is not associated with a predisposition to infection, selection of the dosage and the window of treatment are critical to strike a balance between inflammation and protective immune response to SARS-CoV-2. We believe that early treatment with abatacept might not benefit COVID-19 patients as it would curtail effective protective immune responses against SARS-CoV-2. For the initial exploration, the treatment regimen adapted for the tocilizumab therapy of COVID-19 patients or even the abatacept regimen used in DMARD treatment of rheumatic diseases could be considered.

Emmanuel Stephen-Victor $\odot^{1}$, Mrinmoy Das ${ }^{1}$, Anupama Karnam ${ }^{2}$, Bruno Pitard ${ }^{3}$, Jean-François Gautier ${ }^{2,4}$ and Jagadeesh Bayry $\mathbb{1}^{2}$

${ }^{1}$ Division of Immunology, Boston Children's Hospital, Harvard Medical School, Boston, MA, USA. ${ }^{2}$ Institut National de la Santé et de la Recherche Médicale; Centre de Recherche des Cordeliers, Sorbonne Université, Université de Paris, Paris, France. ${ }^{3}$ Université de Nantes, CNRS ERL6001, Inserm 1232, CRCINA, Nantes, France. ${ }^{4}$ Service de Diabétologie, Endocrinologie, Nutrition, Centre Universitaire du Diabète et de ses Complications, Hôpital Lariboisière, Université Paris-Diderot Paris-7, Paris, France.

Correspondence: Jagadeesh Bayry, Institut National de la Santé et de la Recherche Médicale Unité 1138, Centre de Recherche des Cordeliers, 15 rue de l'Ecole de Médicine, Paris, F-75006, France. E-mail: jagadeesh.bayry@crc.jussieu.fr

Received: 5 June 2020 | Accepted after revision: 23 June 2020

Conflict of interest: E. Stephen-Victor has nothing to disclose. M. Das has nothing to disclose. A. Karnam has nothing to disclose. B. Pitard has nothing to disclose. J-F. Gautier has nothing to disclose. J. Bayry reports grants from Agence 
Nationale de la Recherche, France (Appel Flash COVID-19-COVIMUNE and ANR-19-CE17-0021(BASIN)), outside the submitted work.

Support statement: Partly supported by grants from Agence Nationale de la Recherche, France (Appel Flash COVID-19-COVIMUNE and ANR-19-CE17-0021(BASIN)). Funding information for this article has been deposited with the Crossref Funder Registry.

\section{References}

1 Huang C, Wang Y, Li X, et al. Clinical features of patients infected with 2019 novel coronavirus in Wuhan, China. Lancet 2020; 395: 497-506.

2 Lythgoe MP, Middleton P. Ongoing clinical trials for the management of the COVID-19 pandemic. Trends Pharmacol Sci 2020; 41: 363-382.

3 Bonam SR, Kaveri SV, Sakuntabhai A, et al. Adjunct immunotherapies for the management of severely ill COVID-19 patients. Cell Rep Med 2020; 1: 100016.

4 Wing JB, Tanaka A, Sakaguchi S. Human FOXP3(+) regulatory $\mathrm{T}$ cell heterogeneity and function in autoimmunity and cancer. Immunity 2019; 50: 302-316.

5 Arpaia N, Green JA, Moltedo B, et al. A distinct function of regulatory T cells in tissue protection. Cell 2015; 162: 1078-1089.

6 Qin C, Zhou L, Hu Z, et al. Dysregulation of immune response in patients with COVID-19 in Wuhan, China. Clin Infect Dis 2020; 71: 762-768.

7 Wang F, Hou H, Luo Y, et al. The laboratory tests and host immunity of COVID-19 patients with different severity of illness. JCI Insight 2020; 5: 137799.

8 Chen G, Wu D, Guo W, et al. Clinical and immunological features of severe and moderate coronavirus disease 2019. J Clin Invest 2020; 130: 2620-2629.

9 Wang W, Su B, Pang L, et al. High-dimensional immune profiling by mass cytometry revealed immunosuppression and dysfunction of immunity in COVID-19 patients. Cell Mol Immunol 2020; 17: 650-652.

10 Kalfaoglu B, Almeida-Santos J, Tye CA, et al. T-cell hyperactivation and paralysis in severe COVID-19 infection revealed by single-cell analysis. bioRxiv 2020; preprint [https://doi.org/10.1101/2020.05.26.1159230].

11 Chu H, Zhou J, Wong BH-Y, et al. Middle east respiratory syndrome coronavirus efficiently infects human primary $\mathrm{T}$ lymphocytes and activates the extrinsic and intrinsic apoptosis pathways. J Infect Dis 2016; 213: 904-914.

12 Anghelina D, Zhao J, Trandem $\mathrm{K}$, et al. Role of regulatory $\mathrm{T}$ cells in coronavirus-induced acute encephalitis. Virology 2009; 385: 358-367.

13 Feuerer M, Herrero L, Cipolletta D, et al. Lean, but not obese, fat is enriched for a unique population of regulatory T cells that affect metabolic parameters. Nat Med 2009; 15: 930-939.

14 Wagner N-M, Brandhorst G, Czepluch F, et al. Circulating regulatory t cells are reduced in obesity and may identify subjects at increased metabolic and cardiovascular risk. Obesity (Silver Spring) 2013; 21: 461-468.

15 Bluestone JA, Buckner JH, Fitch M, et al. Type 1 diabetes immunotherapy using polyclonal regulatory T cells. Sci Transl Med 2015; 7: 315ra189.

16 Romano M, Fanelli G, Albany CA, et al. Past, present, and future of regulatory $\mathrm{T}$ cell therapy in transplantation and autoimmunity. Front Immunol 2019; 10: 43.

17 Gladstone DE, Kim BS, Mooney K, et al. Regulatory T cells for treating patients with COVID-19 and acute respiratory distress syndrome: two case reports. Ann Intern Med 2020; in press [DOI: 10.7326/L20-0681]

18 Hartemann A, Bensimon G, Payan CA, et al. Low-dose interleukin 2 in patients with type 1 diabetes: a phase 1/2 randomised, double-blind, placebo-controlled trial. Lancet Diabetes Endocrinol 2013; 1: 295-305.

19 Trotta E, Bessette PH, Silveria SL, et al. A human anti-IL-2 antibody that potentiates regulatory T cells by a structure-based mechanism. Nat Med 2018; 24: 1005-1014.

20 Webster KE, Walters S, Kohler RE, et al. In vivo expansion of T reg cells with IL-2-mAb complexes: induction of resistance to EAE and long-term acceptance of islet allografts without immunosuppression. J Exp Med 2009; 206: 751-760.

21 Qureshi OS, Zheng Y, Nakamura K, et al. Trans-endocytosis of CD80 and CD86: a molecular basis for the cell-extrinsic function of CTLA-4. Science 2011; 332: 600-603.

22 Bonelli M, Scheinecker C. How does abatacept really work in rheumatoid arthritis? Curr Opin Rheumatol 2018 ; 30: 295-300.

23 Bonelli M, Göschl L, Blüml S, et al. Abatacept (CTLA-4Ig) treatment reduces T cell apoptosis and regulatory T cell suppression in patients with rheumatoid arthritis. Rheumatology (Oxford) 2016; 55: 710-720.

24 Langdon K, Haleagrahara N. Regulatory T-cell dynamics with abatacept treatment in rheumatoid arthritis. Int Rev Immunol 2018; 37: 206-214.

25 Michelena X, Borrell H, López-Corbeto M, et al. Incidence of COVID-19 in a cohort of adult and paediatric patients with rheumatic diseases treated with targeted biologic and synthetic disease-modifying anti-rheumatic drugs. Semin Arthritis Rheum 2020; 50: 564-570.

26 Fernandez-Gutierrez B, Leon L, Madrid A, et al. Hospital admissions in inflammatory rheumatic diseases during the COVID-19 pandemic: incidence and role of disease modifying agents. medRxiv 2020; preprint [https://10. 1101/2020.05.21.20108696].

27 Conticini E, Bargagli E, Bardelli M, et al. COVID-19 pneumonia in a large cohort of patients treated with biological and targeted synthetic antirheumatic drugs. Ann Rheum Dis 2020; in press [https://doi.org/10.1136/ annrheumdis-2020-217681].

28 Genovese MC, Becker JC, Schiff M, et al. Abatacept for rheumatoid arthritis refractory to tumor necrosis factor $\alpha$ inhibition. N Engl J Med 2005; 353: 1114-1123. 